\title{
Clinical and visual outcomes of patients with uveitis in the mid-Atlantic United States
}

\author{
This article was published in the following Dove Press journal: \\ Clinical Ophthalmology \\ 8 September 2015 \\ Number of times this article has been viewed
}

\author{
Asima Bajwa' \\ Chang Sup Lee' \\ Jim Patrie ${ }^{2}$ \\ Wenjun Xin ${ }^{2}$ \\ Ashvini K Reddy' \\ 'Department of Ophthalmology, \\ ${ }^{2}$ Department of Public Health \\ Sciences, University of Virginia, \\ Charlottesville, VA, USA
}

Correspondence: Ashvini K Reddy

Department of Ophthalmology,

University of Virginia, 1300 Jefferson Park

Avenue, Charlottesville, VA 22908, USA

Tel +l 4342435890

$\mathrm{Fax}+\mathrm{I} 4349245180$

Email ash.vee.knee@gmail.com
Purpose: To report the clinical outcomes of uveitis patients at the University of Virginia Methods: Retrospective, observational study of uveitis patients seen at the University of Virginia from 1984 to 2014. Parametric and nonparametric methods were used to analyze the change in best-corrected visual acuity (BCVA) in relation to demographics, diagnoses, management, and complications.

Results: The study included 644 eyes of 491 patients. Patients with mild visual loss $(\log$ MAR $<0.4)$ at presentation were younger than those with severe visual loss (SVL, $\log$ MAR $>1.0)(P=0.002)$. Females were more likely to have mild visual loss as compared to males $(P=0.025)$. Median overall BCVA was $\log$ MAR 0.18 at initial and final presentation $(P=1.00)$. Vision loss at diagnosis was a predictor for moderate visual loss (MVL, $\log$ MAR 0.4 to $<1.0)$ to SVL at last follow-up $(P<0.001)$. Eyes with ocular hypertension were positively associated with MVL and SVL as compared to normotensive eyes (1.89 times at baseline, 2.62 times at last follow-up). Median BCVA was $0.18 \log$ MAR for the anterior uveitis (AU) and $0.48 \log$ MAR for the non-AU patients $(P<0.001)$. AU patients were less likely to have SVL than non-AU group $(P<0.001)$. AU group received local corticosteroids more frequently and systemic corticosteroids less commonly than non-AU patients $(P<0.001)$. AU patients with MVL to SVL were more likely to have ophthalmic surgery (cataract, glaucoma or pars plana vitrectomy [PPV]) than those without MVL or SVL $(P<0.001)$. Non-AU patients with MVL to SVL were more likely to have PPV than those without MVL or SVL $(P=0.001)$.

Conclusion: Mean overall BCVA remained stable. Favorable visual results were associated with younger age, female gender, and AU. Poor visual prognosis was concomitant with SVL at presentation and ocular hypertension. Ocular surgery (cataract extraction and glaucoma filtration) was more frequently performed for AU patients with MVL to SVL than those AU patients who did not experience moderate to SVL. PPV was commonly performed for both AU and non-AU patients with MVL to SVL.

Keywords: uveitis, visual outcome, best corrected visual acuity, clinical outcome, visual loss

\section{Introduction}

Uveitis is a term used to describe a group of intraocular inflammatory diseases that can occur at any age but that disproportionately affects patients in the working age group resulting in serious impact on their individual lives and on the society as a whole., It is a relatively uncommon condition with an estimated incidence of 17.4-52.4 cases per 100,000 person-years and a prevalence of $58.0-114.5$ per 100,000 persons. $^{3-7}$ It causes $2.8 \%-10 \%$ of legal blindness in the United States or nearly 30,000 new cases of blindness each year. ${ }^{4}$

Although uveitis is a well-known cause of blindness, there is only limited knowledge concerning the prevalence and incidence of uveitis among the blind. In most 
epidemiological studies dealing with blindness, uveitis is not considered a distinct entity. ${ }^{8-10}$ Previous clinical studies confirm a significant rate of visual impairment due to numerous causes, including cataract, glaucoma, and cystoid macular edema. ${ }^{2,11-13}$ The aims of this retrospective, observational study were to investigate the degree and causes of visual loss in uveitis patients in relation to the development of complications while undergoing treatment.

\section{Methods}

\section{Patient selection}

This was a retrospective observational study of all patients seen in the eye clinic at the University of Virginia, between 1984 and 2014. The study received institutional review board approval. Patient consent was not required as this is a retrospective chart review. An electronic chart review was performed on 1,238 patients with uveitis identified by International Classification of Disease, 9th revision codes corresponding to ocular inflammatory conditions. Individual charts were reviewed and 747 patients were excluded because a diagnosis of uveitis meeting International Uveitis Study Group ${ }^{14,15}$ criteria could not be confirmed by an attending physician (AKR). A further 33 patients (40 eyes) were excluded from the clinical outcome analysis component of the study because no follow-up visit information was available. The total number of patients included in the study was 491 (644 eyes). After excluding 33 patients (40 eyes), best-corrected visual acuity (BCVA) and intraocular pressure (IOP) analysis was done for 458 patients (604 eyes) as the initial and final data was available for this subset of patients only.

\section{Data collection}

For every patient, information was gathered regarding demographic details as well as disease anatomic classification according to the Standardization of Uveitis Nomenclature Working Group criteria, which was categorized as either anterior uveitis (AU) or non-AU (intermediate uveitis, posterior uveitis, and panuveitis) ${ }^{15}$ laterality, cause, and concomitant systemic diseases. We recorded BCVA, IOP, and clinical examination findings for the initial and final visits. The BCVA results were converted to $\log$ MAR units for analysis. ${ }^{16,17}$ Mild or no visual loss (MdVL) was defined as $>20 / 50$ on Snellen chart (logMAR $<0.4)$, moderate visual loss (MVL) as 20/50-20/200 (logMAR 0.4 to <1.0), and severe visual loss (SVL) as $<20 / 200(\log$ MAR $\geq 1.0) .{ }^{15}$ World Health Organization (WHO) definition of blindness (from no light perception to 20/400 logMAR > 1.3) was taken into consideration. ${ }^{18}$ Ocular hypertension was defined as an IOP $>21 \mathrm{mmHg}$ and hypotension as IOP $\leq 7 \mathrm{mmHg} .{ }^{19,20}$
Optical coherence tomography, fluorescein angiography, automated perimetry, and other ancillary tests including serology, radiology, microbiology, and biopsy were performed when appropriate. Intraocular infection was confirmed with fluid sampling or biopsy for microscopy and cytology and culture or polymerase chain reaction, when clinically appropriate. Postprocedural uveitis was defined as ocular inflammation following intraocular surgery, laser, or intravitreal injection. The term "undifferentiated uveitis" was applied if intraocular inflammation could not be attributed to a recognized uveitic entity.

Details of management including the use of local and systemic steroids, intravitreal injections, subtenon injections, antimetabolites, anti-tumor necrosis factor agents, cataract surgery, pars plana vitrectomy (PPV), and glaucoma management (medical and surgical intervention) were recorded. Finally, we collected information regarding burden of disease, including number of clinic visits.

In general, treatment decisions were based on the presence of ocular inflammation as well as associated problems, such as increased IOP and cystoid macular edema. The use of systemic steroids and the addition of second-line immunosuppressive or biologic agents were decided according to clinical judgment of disease activity. Local treatment, using periocular and intravitreal steroid injections was used to reduce the need for systemic drugs and their related side effects.

\section{Statistical analysis Descriptive statistics}

Continuous scaled data were either summarized by the mean and standard deviation of the empirical distribution, or summarized by the median and interquartile range [IQR] of the empirical distribution. Categorical scaled data were summarized by frequencies and percentages.

\section{Visual outcomes}

BCVA in logMAR at baseline and last-follow-up were compared by way of paired permutation tests, in which the null hypothesis was that the underlying distribution for the baseline to last-follow-up change in $\log$ MAR was equal to zero. ${ }^{21}$ A two sided $P \leq 0.05$ decision rule was utilized as the null hypothesis rejection criterion.

McNemar tests were used to compare within each baseline vision loss category (ie, MdVL, MVL, and SVL), the percentage of eyes that were in each category at baseline to the percentage of eyes at last follow-up. A two sided $P \leq 0.05$ decision rule was utilized as the null hypothesis rejection criterion. 
Quantile regression was used to compare the linear trajectories for median logMAR between $\mathrm{AU}$ and non-AU subgroups. ${ }^{22}$ Since several patients had bilateral uveitis, the regression analyses were conducted at the patient level. The $\log$ MAR for bilateral uveitis cases represented the average $\log$ MAR of the two affected eyes.

A binomial multivariate generalized estimating equation model $^{23}$ was utilized to examine the relationship between categories of vision loss (MdVL, MVL, and SVL) at last follow-up and underlying factors predictive of visual morbidity in uveitis. These factors included: patient age at diagnosis, patient gender, race, uveitis laterality (unilateral vs bilateral), baseline vision classification (ie, logMAR $<0.40, \log$ MAR [0.40 to $<1]$, and $\log M A R \geq 1.0$ ), follow-up duration (years), and uveitis location (ie, anterior, intermediate, posterior, or panuveitis), and systemic involvement (ie, yes vs no). Bilateral case outcomes were not assumed to be statistically independent and to account for this nonindependence between outcomes the variance covariance matrix of the generalized estimating equation model was estimated via the working independence sandwich estimator. ${ }^{21}$ Hypothesis testing was based on the Type III Wald statistic, and a two-sided $P \leq 0.05$ decision rule was utilized as the null hypothesis rejection criterion.

\section{IOP outcomes}

McNemar tests were conducted to compare the baseline and follow-up frequencies of ocular hypertension and hypotony. A two-sided $P \leq 0.05$ decision rule was utilized as the null hypothesis rejection criterion.

\section{Treatment differences between $A U$ and non-AU groups}

AU vs non-AU comparisons of medication and surgery requirements were based on the Fisher's exact test. A twosided $P \leq 0.05$ decision rule was utilized as the null hypothesis rejection criterion for all tests.

\section{Statistical software}

The software of SAS version 9.4 (SAS Institute Inc., Cary, NC, USA) and the software of Spotfire S+ (TIBCO Software Inc., Palo Alto, CA, USA) were used to conduct the aforementioned statistical analyses.

\section{Results}

Four-hundred and ninety one patients (644 eyes) were included in this study. A total of $278(56.6 \%)$ patients were females with female: male ratio of 1.3:1. Mean duration of follow-up was 4.8 years $( \pm 6.8)$. Mean number of visits to the eye clinic was $11( \pm 14.3)$. Of the 491 patients, 338 (68.8\%) had unilateral disease. Of the 491 patients, 57 (11.6\%) had documentation of acute onset, and 158 (32.8\%) had insidious onset. A total of 276 (56.2\%) patients had chronic uveitis. Clinical (BCVA, IOP) outcomes were analyzed for only those 458 patients and 604 eyes for whom initial and final BCVA and IOP data were available.

\section{Patient demographics \\ Age}

Different age groups with MdVL, MVL, and SVL at presentation and at the end of the study are given in Table 1 . Overall, the mean age at presentation was $45.5 \pm 21.3$ years (range $0.5-110.6$ years), while the mean age at diagnosis was $46.0 \pm 21.4$ years (range $0.5-110.6$ years). Only $4(0.8 \%)$ patients were more than 100 years of age. For those patients who had BCVA measured at diagnosis $(n=480)$, the mean age at diagnosis was $43.3 \pm 20.3$ years for the cohort of patient who had MdVL at presentation ( $\mathrm{n}=280)$ and $48.7 \pm 21.9$ years for the cohort of patient who had MVL at presentation $(\mathrm{n}=85)$ and 51.2 \pm 23.2 years for the cohort of patients who had SVL at presentation $(n=115)$. Again, the cohort of patients who had MdVL at presentation was somewhat younger than the cohort of subjects with MVL $(P=0.059)$ and SVL $(P=0.002)$ at presentation.

Table I Age groups with MdVL, MVL, and SVL at presentation and at the end of the study

\begin{tabular}{|c|c|c|c|c|c|}
\hline Assessment & $\begin{array}{l}\text { Age } \\
\text { group }\end{array}$ & $\begin{array}{l}\text { Number of eyes (ne), } \\
\text { n (\% overall) }\end{array}$ & $\begin{array}{l}\text { MdVL, } \mathbf{n}(\% \text { within } \\
\text { age group) }\end{array}$ & $\begin{array}{l}\text { MVL, } n \text { (\% within } \\
\text { age group) }\end{array}$ & $\begin{array}{l}\text { SVL, } n \text { (\% within } \\
\text { age group) }\end{array}$ \\
\hline \multirow[t]{4}{*}{ Presentation (ne $=633$ ) } & $<18$ & $54(8.5)$ & $40(69.0)$ & $7(12.1)$ & II (19.0) \\
\hline & $18-35$ & 161 (25.4) & II 8 (73.3) & $12(7.5)$ & $31(19.3)$ \\
\hline & $18-65$ & $448(70.8)$ & $306(68.3)$ & $43(9.9)$ & $99(22.1)$ \\
\hline & $>65$ & $126(20.0)$ & $69(54.8)$ & $14(\mid 1.1)$ & $43(34.1)$ \\
\hline \multirow[t]{4}{*}{ Follow-up (ne =603) } & $<18$ & $56(9.3)$ & $42(75.0)$ & $7(12.5)$ & $7(12.5)$ \\
\hline & $18-35$ & $150(24.9)$ & I $28(85.3)$ & $8(5.3)$ & $14(9.3)$ \\
\hline & $18-65$ & 429 (7I.I) & $330(76.9)$ & $42(9.8)$ & $57(13.3)$ \\
\hline & $>65$ & $123(20.4)$ & $76(61.3)$ & $20(16.3)$ & $27(22.0)$ \\
\hline
\end{tabular}

Notes: $<18$ years: pediatric group; 18-35 years: young adults; 18-65 years: working age group; $>65$ years: older age group.

Abbreviations: MdVL, mild visual loss; MVL, moderate visual loss; SVL, severe visual loss. 


\section{Gender}

There were 278 female patients (56.6\%). Within the MdVL cohort, there were 167 (59.6\%), while within the MVL cohort there were 46 (54.1\%) female patients, and within the SVL cohort there were 54 (50.9\%) female patients. Females were more likely to have MdVL at presentation than to have SVL at presentation, where the converse was true for males $(P=0.025)$. Baseline characteristics of the uveitis patient at the time of diagnosis are presented in Table 2.

\section{Follow-up}

The median length of follow-up was 2.0 years (IQR: $0.2-6.8$ years, range $0-30$ years, mean 4.8 years), 1,948 patient-years and 2,475 eye-years. Sixty-six eyes (16.2\%) had more than 10 years follow-up, and the median follow-up for those eyes was 15.7 years with range $10.2-30$ years.

Table 2 Baseline characteristics of the uveitis patient at the time of diagnosis

\begin{tabular}{|c|c|}
\hline & Number (\%) \\
\hline \multicolumn{2}{|l|}{ Patient level baseline characteristics } \\
\hline Number of patients (n) & 491 \\
\hline Female gender & $278(56.6)$ \\
\hline Mean age (SD), years & $45.5(21.5)$ \\
\hline \multicolumn{2}{|l|}{ Race } \\
\hline Asian & $5(1.0)$ \\
\hline African American & I $34(27.3)$ \\
\hline Caucasian & $298(60.7)$ \\
\hline Hispanic & $12(2.4)$ \\
\hline Other & $42(8.5)$ \\
\hline Systemic involvement & $335(68.2)$ \\
\hline Bilateral vision reported $(n=49 I)$ & $153(3 \mid .2)$ \\
\hline \multicolumn{2}{|l|}{ Vision loss $(n=48 I)$} \\
\hline MdVL (logMAR: <0.4) & $280(58.2)$ \\
\hline MVL (logMAR: [0.4-I]) & $85(17.7)$ \\
\hline SVL (logMAR: $\geq 1$ ) & $116(24.1)$ \\
\hline \multicolumn{2}{|l|}{ Eye level baseline characteristics } \\
\hline Number of eyes (ne) & 644 \\
\hline Non-anterior uveitis & $237(36.8)$ \\
\hline Median length of follow-up & $4.8(0.2-6.8)$ \\
\hline \multicolumn{2}{|l|}{ (interquartile range) years, $(\mathrm{ne}=520)$} \\
\hline Mean BCVA (SD), logMAR (ne =633) & $0.52(0.74)$ \\
\hline \multicolumn{2}{|l|}{ Visions loss ( $\mathrm{ne}=633$ ) } \\
\hline MdVL (logMAR: <0.4) & $415(65.6)$ \\
\hline MVL (logMAR: [0.4-I]) & $64(10.1)$ \\
\hline SVL $(\log M A R: \geq 1)$ & $154(24.3)$ \\
\hline Mean BCVA overall (SD), logMAR & $0.52(0.74)$ \\
\hline \multicolumn{2}{|l|}{$(n e=633)$} \\
\hline MdVL, mean BCVA (SD) & $0.13(0.13)$ \\
\hline MVL, mean BCVA (SD) & $0.56(0.06)$ \\
\hline$S V L$, mean BCVA (SD) & $1.56(0.86)$ \\
\hline
\end{tabular}

Abbreviations: BCVA, best-corrected visual acuity; logMAR, logarithm of the minimum angle of resolution; SD, the standard deviation of the measurement distribution; MdVL, mild visual loss; MVL, moderate visual loss; SVL, severe visual loss; ne, number of eyes.

\section{Visual outcome}

With respect to the entire group of uveitis patients ( $n=458$; 604 eyes) who had BCVA assessed at baseline and then at last follow-up, the median BCVA at the time of diagnosis was $0.18 \log$ MAR (IQR: $0.10-0.60 \log M A R$ ), and the final median BCVA at the end of the study was $0.18 \log$ MAR (IQR: $0.0-0.48 \log M A R$ ).

At baseline ( $\mathrm{n}=604$ eyes), there were $400(66.2 \%)$ eyes from 322 patients $(70.3 \%)$ with MdVL ( $20 / 40$ or better, $<0.4$ $\log$ MAR), 62 eyes (10.3\%) from 56 patients (13.5\%) with MVL (20/40-20/200, logMAR 0.4-1), and 142 eyes (23.5\%) from 133 patients $(29.0 \%)$ with SVL (20/200 logMAR 1.0 or worse). At the end of the study ( $n=604$ eyes), there were 444 (73.5\%) eyes from 351 (76.6\%) patients with MdVL, 69 (11.4\%) eyes from 64 (10.6\%) patients with MVL, and 91 (15.0\%) eyes from 87 (14.4\%) patients with SVL. Of the 400 eyes with MdVL at presentation, 15 (3.7\%) eyes progressed to MVL by last-follow-up while 35 (8.7\%) eyes progressed to SVL. Of the 62 eyes with MVL at diagnosis, 43 (69.4\%) improved to MdVL by last-follow-up, while 11 (17.7\%) eyes progressed to SVL. Of the 142 eyes with SVL at diagnosis, $51(35.9 \%)$ eyes improved to MdVL by last-follow-up, while 10 (7.0\%) eyes improved to MVL. A tabular summary of the visual outcome information is provided in Table 3, with additional information pertaining BCVA within the three vision loss subgroups, and the baseline to follow-up changes in BCVA are graphically presented in Figure 1. At the end of the study, a total of 73 (12.1\%) eyes met WHO criteria for blindness. Twenty-two patients had bilateral blindness.

\section{Vision stability:AU vs non-AU}

Eye level analyses revealed that at the time of diagnosis, median $\log$ MAR was 0.18 (95\% CI: [0.14, $0.21 \log \mathrm{MAR}]$ ) for the AU patients and 0.30 units (95\% CI: [0.22, 0.38 logMAR]) for the non-AU patients $(P=0.008)$. The predicted median $\log$ MAR trajectories for AU and non-AU eyes are displayed in Figure 2. For AU eyes, the follow-up trajectory for median $\log$ MAR was $0.007 \log$ MAR units/year $(95 \% \mathrm{CI}$ : $[0.000,0.013 \log \mathrm{MAR} / \mathrm{year}], P=0.034)$, while for the non-AU patients, the follow-up trajectory for median logMAR was $0.004 \log$ MAR units/year (95\% CI: [-0.000, $0.015 \log$ MAR/ year], $P=0.064)$. The median trajectories were not statistically different between the two groups $(P=0.877)$.

Patient level analyses revealed that at the time of diagnosis, median $\log$ MAR was 0.18 (95\% CI: [0.13, 0.23 $\log \mathrm{MAR}])$ for the $\mathrm{AU}$ patients and 0.48 units (95\% CI: [0.38, $0.57 \log \mathrm{MAR}])$ for the non-AU patients $(P<0.001)$. For $\mathrm{AU}$ 
Table 3 Visual outcomes for the subset of uveitis patients with both baseline and follow-up visual acuity assessments ( $\mathrm{n}=458 ; 604$ eyes)

\begin{tabular}{llll}
\hline Vision group at baseline & Baseline assessment & Follow-up assessment & P-value* \\
\hline **logMAR, median (IQR) & $0.18(0.10-0.60)$ & $0.18(0.0-0.48)$ & 1.000 \\
MdVL, median (IQR) & $0.10(0.00-0.18)$ & $0.10(0.0-0.30)$ & 1.000 \\
MVL, median (IQR) & $0.48(0.48-0.60)$ & $0.30(0.11-0.48)$ & $<0.00 \mathrm{I}$ \\
SVL, median (IQR) & $1.18(1.00-1.47)$ & $1.00(0.30-1.30)$ & $<0.00 \mathrm{I}$ \\
MdVL, ne (\%) & $400(66.2)$ & $444(70.1)$ & $<0.00 \mathrm{I}$ \\
MVL, ne (\%) & $62(10.3)$ & $69(10.9)$ & 0.468 \\
SVL, ne (\%) & $142(23.5)$ & $9 \mathrm{I}(14.4)$ & $<0.00 \mathrm{I}$ \\
\hline
\end{tabular}

Notes: *P-value based on a permutation test, where the null hypothesis was that the difference between the baseline and the follow-up median logMAR is simply a consequence of chance; **all visual loss cohorts combined.

Abbreviations: logMAR, logarithm of the minimum angle of resolution; MdVL, mild visual loss; MVL, moderate visual loss; ne, number of eyes; SVL, severe visual loss.

patients, the follow-up trajectory for median $\log$ MAR was 0.008 logMAR units/year (95\% CI: [ $-0.000,0.017$ logMAR/ year], $P=0.066$ ), while for the non-AU patients, the follow-up trajectory for median logMAR was $0.004 \operatorname{logMAR}$ units/ year (95\% CI: [-0.014, $0.023 \log$ MAR/year], $P=0.636)$. The median trajectories were not statistically different between the two groups.

Eyes with AU ( $n=386)$ vs eyes with non-AU $(n=223)$ were no more likely to have MVL (11.9\% vs $10.3 \%$ respectively, $P=0.571)$. Eyes with $\mathrm{AU}$, however, were less likely ( $10.6 \%$ vs $22.4 \%$, respectively) to have SVL (odds ratio 2.43 ; $95 \%$ CI: $[1.52,3.89], P<0.001)$.

\section{Diagnosis}

The anatomical classification for the cohort is given in Table 4.
The relative frequencies of key uveitis diagnoses and the number of eyes with MdVL, MVL, and SVL at the end of study are given in Table 5 .

\section{Factors predictive of visual morbidity in uveitis}

Multivariate analyses showed that for the 500 eyes that had complete baseline demographics and diagnostic information, neither age at uveitis diagnosis $(P=0.089)$, nor gender $(P=0.264)$, nor race $(P=0.144)$ were predictors of MVL or SVL at last follow-up. Similarly, neither follow-up duration $(P=0.675)$, nor uveitis laterality status $(P=0.359)$ were predictors of MVL or SVL at last follow-up. The location of uveitis at diagnosis was also not a predictor of MVL or SVL at last follow-up $(P=0.359)$, nor was systemic involvement $(P=0.371)$. However, vision loss at diagnosis was a predictor for MVL or SVL at last follow-up $(P<0.001)$. Eyes that

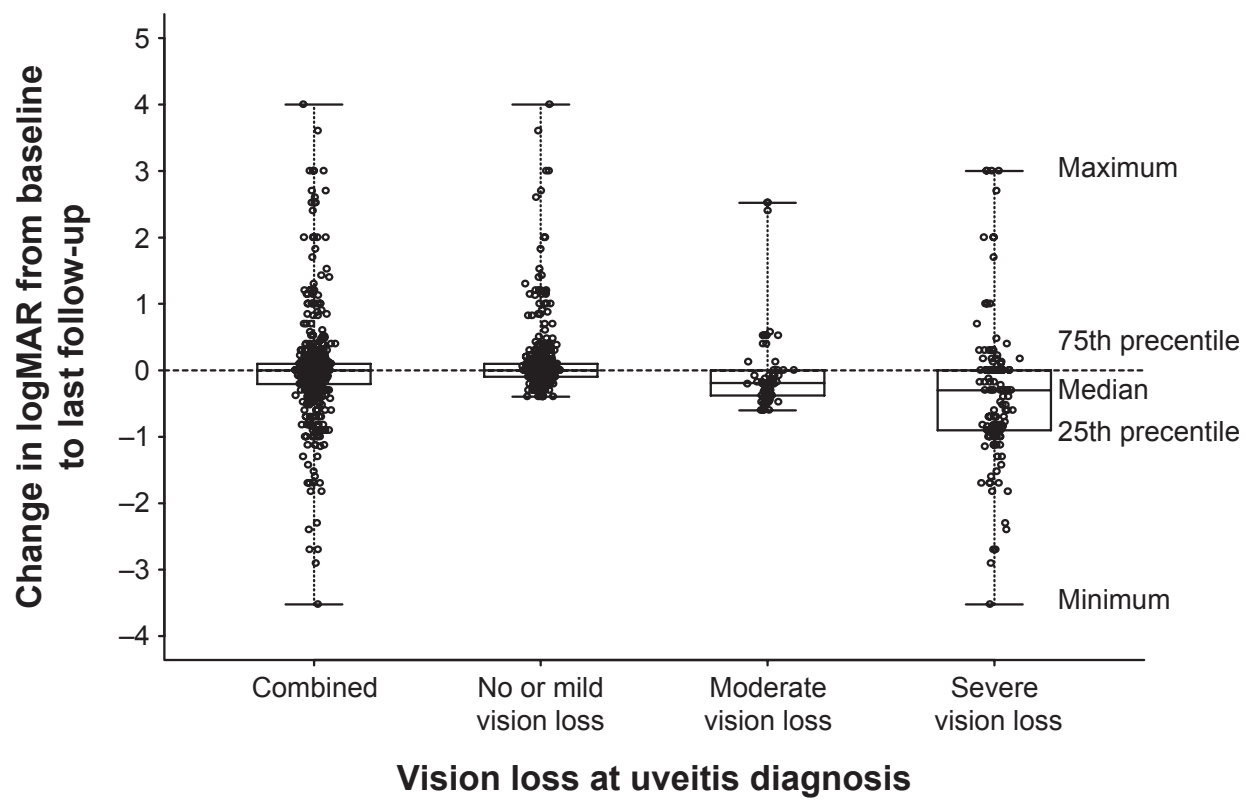

Figure I Change in logMAR at last follow-up from logMAR at baseline (ie, at diagnosis) for the set of eyes that had both baseline and follow-up BCVA assessed ( $\mathrm{n}=604$ eyes). Note: Hatch horizontal line identifies zero change in logMAR.

Abbreviations: BCVA, best-corrected visual acuity; logMAR, logarithm of the minimum angle of resolution. 


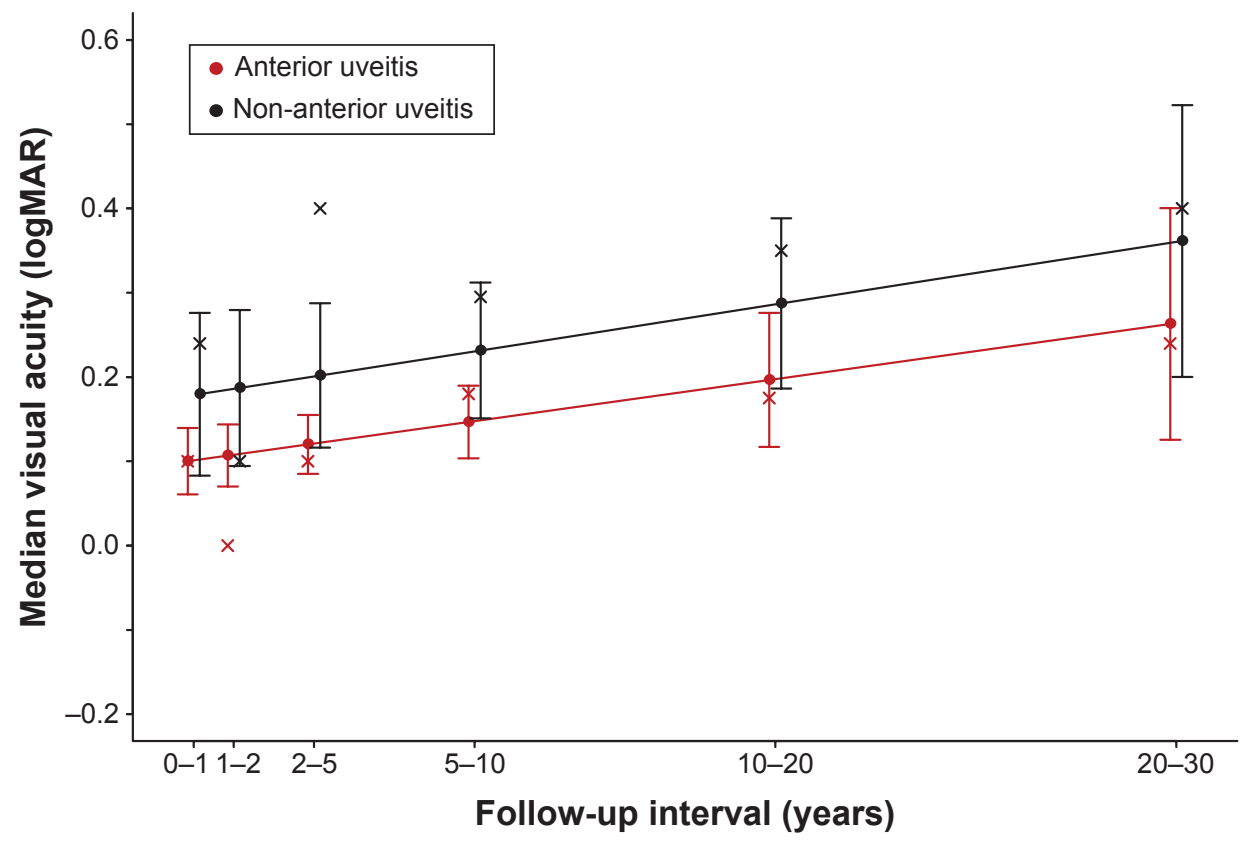

Figure 2 Quantile regression predictions for patient level median logMAR as a linear function of follow-up time (years).

Note: Solid points identify the predicted median logMAR at the midpoint of the time interval and the " $x$ " symbol identifies the empirical median logMAR for the time interval. Abbreviation: logMAR, logarithm of the minimum angle of resolution.

had MVL at the time of uveitis diagnosis were more likely (odds ratio 3.05 ; 95\% CI: $[1.50,6.16]$ ) to have MVL to SVL at follow-up than eyes that had MdVL at uveitis diagnosis $(P=0.002)$. Eyes that had SVL at the time of uveitis diagnosis, were considerably more likely (odds ratio $9.57,95 \% \mathrm{CI}$ : [5.73, 15.96], $P<0.001$ ) to have MVL to SVL at last follow-up than eyes with MdVL at uveitis diagnosis. Eyes that had SVL at the time of uveitis diagnosis, were also considerably more likely (odds ratio $3.14,95 \% \mathrm{CI}$ : $[1.53,6.46], P=0.002$ ) than eyes with MVL at diagnosis to have MVL to SVL at last follow-up.

\section{IOP}

Of the 644 eyes from 491 patients, 618 (96.0\%) eyes from 471 patients had baseline IOP recorded. Median initial IOP was $15.0 \mathrm{mmHg}$ (IQR: 12, $18 \mathrm{mmHg}$ ). Seventy-six (12.3\%) eyes from 68 patients presented with ocular hypertension at baseline. Six-hundred and five (93.4\%) eyes from 459 patients had IOP assessed at last follow-up. Median final IOP was
$14.0 \mathrm{mmHg}$ (IQR: $12-18 \mathrm{mmHg}$ ). Forty-seven (7.8\%) eyes from 46 patients had ocular hypertension at follow-up.

At both the baseline and the follow-up assessments, ocular hypertension was positively associated with MVL and SVL while hypotony was not. If an eye was hypertensive at the baseline assessment, the odds that the eye had MVL to SVL was 1.89 times $(95 \%$ CI: $[1.15,3.11])$ than the odds for an eye that was normotensive $(P=0.012)$. If an eye was hypertensive at follow-up, the odds that the eye had MVL to SVL was 2.62 time ( $95 \%$ CI: [1.38, 4.98], $P=0.003$ ) than the odds for an eye that was normotensive.

\section{Treatment of uveitis, complications and interventions} Management of uveitis patients is documented in Table 6.

\section{Treatment differences between $A U$ and non-AU}

There was no significant difference in the median annual number of clinical visits between AU and non-AU patients

Table 4 Anatomical classification ( $n=49$ I, 644 eyes)

\begin{tabular}{llll}
\hline Classification & $\begin{array}{l}\text { Number of } \\
\text { patients (\%) }\end{array}$ & $\begin{array}{l}\text { Number of patients with } \\
\text { bilateral disease (\%) }\end{array}$ & $\begin{array}{l}\text { Number of patients with } \\
\text { unilateral disease (\%) }\end{array}$ \\
\hline $\begin{array}{l}\text { Anterior uveitis } \\
\text { Non-anterior uveitis }\end{array} \quad$ 75 (22.6) & $257(77.4)$ \\
$\quad 332(67.6)$ & $19(73.1)$ & $7(26.9)$ \\
Intermediate uveitis & $26(5.3)$ & $32(51.6)$ & $30(48.4)$ \\
Posterior uveitis & $62(12.6)$ & $27(38.0)$ & $44(62.0)$ \\
$\quad$ Panuveitis & $71(19.5)$ & & \\
\hline
\end{tabular}


Table 5 Diagnostic uveitis classification for MdVL, MVL, SVL for those eyes that had both initial and final BCVA available $(n=458$; 604 eyes)

\begin{tabular}{lll}
\hline $\begin{array}{l}\text { Visual loss } \\
\text { (number of eyes [ne]) }\end{array}$ & Diagnosis & $\begin{array}{l}\text { Number of } \\
\text { eyes* (\%) }\end{array}$ \\
\hline MdVL (ne=444) & Trauma & $48(10.8)$ \\
& Sarcoidosis & $34(7.7)$ \\
AU-HLAB27 & $34(7.7)$ \\
& AU-undifferentiated & $20(29.0)$ \\
SVL (ne=91) & Sarcoidosis & $10(14.5)$ \\
& Postprocedural & $6(8.7)$ \\
& Postprocedural & $16(17.6)$ \\
& AU-undifferentiated & $12(13.2)$ \\
& Trauma & $7(7.7)$ \\
& Sarcoidosis & $7(7.7)$ \\
& Panuveitis-undifferentiated & $7(7.7)$ \\
\hline
\end{tabular}

Note: *Percentages are for each group of disease severity and not for the entire study population.

Abbreviations: AU, anterior uveitis; BCVA, best-corrected visual acuity; HLAB27, human leucocyte antigen B27; MdVL, mild visual loss; MVL, moderate visual loss; SVL, severe visual loss.

(median 6; IQR: 3-13 vs median 6; IQR: 3-15, respectively, $P=1.000)$.

Fewer patients with $\mathrm{AU}(66 / 329,20.1 \%)$ were treated with oral prednisone $(P<0.001)$ than those with non-AU (67/159, 42.1\%). More AU patients (274/331, 82.8\%) received local steroid injections $(P<0.001)$ than patients with non-AU $(91 / 158,57.6 \%)$. The prescription of secondline agents was similar between AU patients (10/330, 3.0\%) and non-AU patients $(7 / 159,4.4 \%)(P=0.439)$.

AU and non-AU patients experiencing MVL to SVL were compared to patients not experiencing MVL to SVL. Among AU patients, those who experienced MVL to SVL were not likely to receive systemic corticosteroids than those who did not experience MVL or SVL (20/79 [25.3\%] vs $33 / 233$ [18.9\%], respectively, $P=0.259$ ). AU patients who experienced MVL to SVL were also no more likely to

Table 6 Ophthalmic management and interventions

\begin{tabular}{ll}
\hline Treatment/intervention & $\begin{array}{l}\text { Number } \\
\text { of patients (\%) }\end{array}$ \\
\hline Local steroids & $365(74.6)$ \\
Systemic steroids & $133(27.3)$ \\
Anti-TNF $\alpha$ agents & $17(3.5)$ \\
Antimetabolites & $52(10.6)$ \\
Intravitreal injection & $54(11.0)$ \\
Subtenon injection & $23(4.7)$ \\
Glaucoma topical treatment & $116(23.6)$ \\
Glaucoma surgery & $43(8.8)$ \\
Cataract surgery & $129(26.4)$ \\
Pars plana vitrectomy & $46(9.4)$ \\
\hline
\end{tabular}

Abbreviation: TNF $\alpha$, tumor necrosis factor alpha. receive a second-line therapeutic agent than those who did not experience MVL to SVL (3/80 [3.7\%] vs 7/233 [3.0\%], respectively, $P=0.720)$. AU patients, who experienced MVL to SVL, however, were more likely to have cataract or glaucoma surgery than those who did not experience MVL to SVL (38/80 [52.5\%] vs 54/179 [23.2\%], respectively, $P<0.001)$, or to have PPV (11/80 [1.37\%] vs 5/228 [2.1\%], respectively, $P<0.002)$.

Among non-AU patients, those who experienced MVL to SVL were not likely to receive systemic corticosteroids than those who did not experience MVL to SVL (27/66 [40.9\%] vs 32/82 [39.0\%], respectively, $P=0.867)$. Non-AU patients who experience MVL to SVL were also not likely to receive a second line therapeutic agent than those who did experience MVL to SVL (2/66 [3.0\%] vs 5/82 [6.1\%], respectively, $P=0.468$ ). Non-AU patients, who experience MVL to SVL, were not likely to have cataract or glaucoma surgery than those who did experience MVL to SVL (16/66 [24.2\%] vs 22/82 [26.8\%], respectively, $P=0.856$ ), but more likely to have PPV (20/66 [30.3\%] vs 7/82 [8.5\%], respectively, $P=0.001)$.

\section{Discussion}

Several factors affect the progress and the outcomes of uveitis. The primary focus of this study was to evaluate the visual and clinical outcomes of uveitis over a period of 30 years.

The average age of diagnosis was 46 years which is comparable to the range reported in other studies (46.1-48 years). ${ }^{12,13,24}$ Patients with SVL at presentation were significantly older than those with MdVL to MVL. A similar observation has been reported previously. ${ }^{13,25}$ This is probably related to an increasing prevalence of panuveitis and bilateral disease with increasing age, though Darrell et al did not find age to be a risk factor. ${ }^{6}$ Tomkins-Netzer et al reported that uveitis occurs in young patients. ${ }^{2}$ Most of the patients included in our analysis were in the working age group as well. Mean duration of follow-up for our study was 4.8 years which is much longer than reported in other studies. ${ }^{12,13}$ Overall, we had more female patients $(56.6 \%)$, which has been reported in earlier studies $(57.8 \%-62 \%) .{ }^{13,24,26}$ As these studies and ours is based on patients from a tertiary referral service, it raises the possibility that women are more susceptible to severe uveitis than men. One of the studies reported that the age of onset of uveitis and patient gender were not found to have a statistically significant impact on vision. ${ }^{2}$ Interestingly, female patients in our study had lower rate of SVL at presentation. It is possible for women being more inclined to 
seek medical attention than men. This has not been reported elsewhere to our knowledge.

Durrani et al reported that patients with bilateral intraocular inflammation, panuveitis, Asian origin, and increasing age had a poorer visual prognosis. ${ }^{13}$ Tomkins-Netzer et al confirmed increased risk for visual loss in patients with non-AU disease, vitreous opacities, retinal detachment, cystoid macular edema, macular scarring, macular hole, optic neuropathy, or macular ischemia. ${ }^{2}$ The multivariate analysis of our study did not support age, gender, ethnic origin, location, laterality of the disease, and follow-up duration as predictors of visual loss. However, we observed that SVL at baseline was significantly associated with MVL to SVL at the end of the study. This would imply the need for early diagnosis and appropriate management.

In our study, $14.4 \%$ of eyes experienced SVL $<20 / 200$ and $70.1 \%$ had visual acuity $>20 / 40$ at the end of the study. Though, this visual loss is in accordance with the earlier report by Durrani et al it is higher than reported in other studies. ${ }^{2,13}$ The overall average visual acuity remained stable over our study period. Approximately $70 \%$ of eyes achieved visual acuity of better than 20/40 and approximately $80 \%$ attained visual acuity of better than $20 / 200$. An earlier study reported that average $\mathrm{BCVA}$ remained stable throughout follow-up for more than 10 years, with approximately $80 \%$ avoiding vision loss. Other studies have shown variable visual outcomes. The percentage of patients avoiding MVL (BCVA > 20/50) ranges from 30\% at an average final follow-up of 36.7 months in a study by Durrani et al to $65 \%$ at 4.3 years in a study by Rothova et al. ${ }^{11,13}$ Bodaghi et al established that at a final average follow-up of 3.5 years, more than $85 \%$ of eyes had a BCVA of $20 / 200$ or better. ${ }^{12}$ In a UK cohort of 315 patients, $69.9 \%$ experienced visual loss ( $<6 / 18$ with $50.45 \%$ having bilateral reduced vision) with $72 \%$ having severe loss of vision. Their percentage was higher than previously reported, ${ }^{1,11,27}$ and it may be due to their definition of visual loss $(<6 / 18) .{ }^{13}$ Most other studies used the WHO definition of vision loss. ${ }^{1,11,28-30}$ By upholding a stratagem of early treatment decisions and immunosuppression to maintain a state of remission, it is possible to maintain a steady BCVA in most patients. ${ }^{27}$

The incidence of blindness among patients with uveitis is poorly defined. The incidence of total blindness due to uveitis in the United States was estimated to be $10 \%-15 \% .{ }^{31}$ Seventy three eyes (12.1\%) in our study group met WHO criteria for blindness which is close to the one observed by Durrani et al (11.4\%). ${ }^{13}$ Twenty-two (4.8\%) of our patients developed bilateral blindness. In another series of 582 patients, bilateral legal blindness developed in 22 (4\%) patients. ${ }^{11}$ Severe uveitis that is resistant to treatment; uveitis that is not promptly and effectively treated; or uveitis that is accompanied by significant secondary complications may result in poor visual outcomes, including blindness. Despite this, with appropriate modern therapy in informed, cooperative patients, few individuals with uveitis should suffer such devastating outcomes.

In our study, ocular hypertension was associated with MVL and SVL. Of interest, the majority (89.7\%) of patients who presented with ocular hypertension at the initiation of the study period were normotensive at the end of the study. Further analysis of ocular hypotony and hypertension as determinants of uveitis outcomes will be revealed in one of our forthcoming papers.

The etiology of uveitis and the anatomical location of uveitis alter the course and the outcome of uveitis. Earlier studies conducted at tertiary referral centers reported that more patients are likely to suffer from bilateral disease and panuveitis. ${ }^{12,13,26}$ Results from community-based studies report $\mathrm{AU}$ as the most common type of uveitis. ${ }^{32,33} \mathrm{In}$ our cohort, AU was the most common anatomical type observed. None of the anatomical uveitis diagnoses were associated with significant visual loss. Further analysis revealed that visual acuity was significantly different between AU and non-AU groups. Non-AU group presented with worse vision than the AU cohort, which has been reported earlier. ${ }^{2}$

SVL was more common among patients with pan-uveitis, followed by AU in a few of the previous reports. ${ }^{11-13}$ In this series, the most common cause of SVL was postprocedural uveitis followed by undifferentiated AU, trauma, and undifferentiated panuveitis. This difference in types of uveitis associated with SVL may be explained by different classification criteria employed. Most of the previous papers used anatomical classification, ${ }^{11-13}$ while we reported both anatomical and etiological categories of uveitis.

Common complications of uveitis include glaucoma and cataract, and the management of those complications could be medical or surgical depending on the severity. Rothova et al stated that $23 \%$ of their 582 patients required one or more intraocular surgical procedures. The percentage of those requiring surgery increased with the duration of follow-up. The most frequent surgical procedures were cataract extractions, vitrectomy, surgery for glaucoma, and retinal detachment. ${ }^{11}$ Approximately one-third of the eyes in our cohort required ocular surgery, with cataract extraction being the most common procedure. In our observation, cataract and 
glaucoma surgery was more frequently performed on patients with AU who suffered MVL to SVL, as also reported by Tomkins-Netzer et al. ${ }^{2}$ Although these procedures are considered low-risk for patients without uveitis, uveitis patients face are at increased risk of complications, including intraocular inflammation. ${ }^{34-36}$ It has been recommended that eyes be quiet for at least three months prior to surgery, thus the patient may suffer prolonged visual morbidity until the eye is optimized for surgery.

In this study, almost $75 \%$ patients received local and $27 \%$ systemic corticosteroids. Fewer patients with AU compared with those with non-AU required systemic corticosteroids but more of them received local corticosteroids. Similar results have been published earlier. ${ }^{2}$ A retrospective study of 834 uveitis cases conducted in Tokyo reported that approximately $60 \%$ of patients received topical and/or periocular injections of corticosteroids; and approximately one-fourth (28.6\%) of patients required some form of systemic immunomodulatory therapy including corticosteroids. ${ }^{24}$

\section{Limitations}

This review is based on data from a tertiary center and is therefore susceptible to limitations based on referral bias, treatment bias, and retrospective data collection. Moreover, management may have been influenced by health insurance policy, the availability of uveitis specialist, and the treatment preferences of the uveitis specialists involved in the study.

\section{Conclusion}

In this series of patients with uveitis, we observed that 1) median BCVA was stable from initial to final visit; 2) poorer visual outcomes were seen in patients with SVL at presentation, non-AU, and ocular hypertension; 3) better visual outcomes were associated in patients with younger age, female gender and AU; 4) approximately two-thirds of patients had no or mild vision loss; 5) approximately onethird of patients required systemic treatment; and 6) less than half of patients required ocular surgery.

\section{Acknowledgments}

The authors would like to thank Irfan Khan, Vandan Patel, and Shuo Qiu for their contribution to data collection. Meeting presentation: a part of this study was presented as a poster at ARVO, June 2015, Denver, CO, USA.

\section{Disclosure}

The authors report no conflicts of interest in this work.

\section{References}

1. Suttorp-Schulten MSA, Rothova A. The possible impact of uveitis in blindness: a literature survey. Br J Ophthalmol. 1996;80:844-888.

2. Tomkins-Netzer O, Talat L, Bar A, et al. Long-term clinical outcome and causes of vision loss in patients with uveitis. Ophthalmology. 2014;121(12):2387-2392.

3. Suhler EB, Lloyd MJ, Choi D, Rosenbaum JT, Austin DF. Incidence and prevalence of uveitis in Veterans Affairs Medical Centers of the Pacific Northwest. Am J Ophthalmol. 2008;146:890-896.

4. Acharya NR, Tham VM, Esterberg E, et al. Incidence and prevalence of uveitis: results from the Pacific Ocular Inflammation Study. JAMA Ophthalmol. 2013;131:1405-1412.

5. Gritz DC, Wong IG. Incidence and prevalence of uveitis in Northern California: the Northern California Epidemiology of Uveitis Study. Ophthalmology. 2004;111:491-500.

6. Darrell RW, Wagener HP, Kurland LT. Epidemiology of uveitis. Incidence and prevalence in a small urban community. Arch Ophthalmol. 1962;68:502-514.

7. Paivonsalo-Hietanen T, Tuominen J, Vaahtoranta-Lehtonen $\mathrm{H}$, Saari KM. Incidence and prevalence of different uveitis entities in Finland. Acta Ophthalmol Scand. 1997;75:76-81.

8. Leibowitz HM, Krueger DE, Maunder LR, et al. The Framingham Eye Study monograph. Surv Ophthalmol (suppl). 1980;24:335-610.

9. Thylefors B, Negrel AD, Pararajasegaram R, Dadzie KY. Global data on blindness. WHO Bulletin. 1995;73:115-121.

10. Tielsch JM, Javitt JC, Coleman A, Katz J, Sommer A. The prevalence of blindness and visual impairment among nursing home residents in Baltimore. N Engl Med. 1995;332:1205-1209.

11. Rothova A, Suttorp-van SMS, Frits TW, Kijlstra A. Causes and frequency of blindness in patients with intraocular inflammatory disease. Br J Ophthalmol. 1996;80:332-336.

12. Bodaghi B, Cassoux N, Wechsler B, et al. Chronic severe uveitis: etiology and visual outcome in 927 patients from a single center. Medicine (Baltimore). 2001;80:263-270.

13. Durrani OM, Tehrani NN, Marr JE, et al. Degree, duration, and causes of visual loss in uveitis. Br J Ophthalmol. 2004;88:1159-1162.

14. Bloch-Michel E, Nussenblatt RB. International uveitis study group recommendations for the evaluation of intraocular inflammatory disease Am J Ophthalmol. 1987;103:234-235.

15. The Standardization of Uveitis Nomenclature (SUN). Working Group. Standardization of Uveitis Nomenclature for Reporting Clinical Data. Results of the First International Workshop. Am J Ophthalmol. 2005;140:509-516.

16. Lange C, Feltgen N, Junker B, Schulze-Bonsel K, Bach M. Resolving the clinical acuity categories "hand motion" and "counting fingers" using the Freiburg Visual Acuity Test (FrACT). Graefes Arch Clin Exp Ophthalmol. 2009;247(1):137-142.

17. Kwon YH, Kim CS, Zimmerman MB, Alward WL, Hayreh SS. Rate of visual field loss and long-term visual outcome in primary open-angle glaucoma. Am J Ophthalmol. 2001;132(1):47-56.

18. McGavin M. Reaching for high standards of eye care. Community Eye Health. 1993;6:1-2.

19. Varma R, Ying-Lai M, Francis BA, et al. Prevalence of open-angle glaucoma and ocular hypertension in Latinos: the Los Angeles Latino Eye Study. Ophthalmology. 2004;111(8):1439-1448.

20. Sen HN, Drye LT, Goldstein DA, et al. Hypotony in patients with uveitis: the Multicenter Uveitis Steroid Treatment (MUST) trial. Ocul Immunol Inflamm. 2012;20(2):104-112.

21. Collingridge DS. A primer on quantized data analysis and permutation testing. J Mix Methods Res. 2013;7(1):81-97.

22. Koenker R. Quantile regression (No 38). Cambridge: Cambridge University Press; 2005.

23. Hardin JW. Generalized estimating equations (GEE). Hoboken, NJ: John Wiley \& Sons, Ltd; 2005.

24. Keino H, Nakashima C, Watanabe T, et al. Frequency and clinical features of intraocular inflammation in Tokyo. Clin Exp Ophthalmol. 2009;37:595-601. 
25. Rodriguez A, Calonge M, Pedroza-Seres M, et al. Referral patterns of uveitis in a tertiary eye care center. Archives of Ophthalmology. 1996; 114(5):593-599.

26. Merrill PT, Kim J, Cox TA, et al. Uveitis in the southeastern United States. Curr Eye Res. 1997;16:865-874.

27. Tomkins-Netzer O, Taylor SR, Lightman S. Long-term clinical and anatomic outcome of birdshot chorioretinopathy. JAMA Ophthalmol. 2014; 132:57-62.

28. Couto C, Merlo JL. Epidemiological study of patients with uveitis in Buenos, Amsterdam Argentina. Recent advances in uveitis. Dernouchamps JP, Verougstaete C, Caspers-velu L, Tassignon MJ, eds. Amsterdam: Kugler Publications, 1993;171-174.

29. Kotaniemi K, Aho K, Kotaniemi A. Uveitis as a cause of visual loss in arthritides and comparable conditions. J Rheumatol. 2001;28: 309-312.

30. Rosner RS. Uveitis and blindness. Med Trial Tech Q. 1967;14: $39-42$.
31. Nussenblatt RB. The natural history of uveitis. Int Ophthalmol. 1990; 14:303-308.

32. McCannel CA, Holland GN, Helm CJ, et al. Causes of uveitis in the general practice of ophthalmology. UCLA Community-Based Uveitis Study Group. Am J Ophthalmol. 1996;121:35-46.

33. Paivonsalo-Hietanen T, Vaahtoranta-Lehtonen H, Tuominen J, et al. Uveitis survey at the University Eye Clinic in Turku. Acta Ophthalmol (Copenh). 1994;72:505-512.

34. Jancevski M, Foster CS. Cataracts and uveitis. Discov Med. 2010; 9:51-54.

35. Siddique SS, Suelves AM, Baheti U, Foster CS. Glaucoma and uveitis. Surv Ophthalmol. 2013;58:1-10.

36. Loewenstein A, Zur D. Postsurgical cystoid macular edema. Dev Ophthalmol. 2010;47:148-159.
Clinical Ophthalmology

\section{Publish your work in this journal}

Clinical Ophthalmology is an international, peer-reviewed journal covering all subspecialties within ophthalmology. Key topics include: Optometry; Visual science; Pharmacology and drug therapy in eye diseases; Basic Sciences; Primary and Secondary eye care; Patient Safety and Quality of Care Improvements. This journal is indexed on

\section{Dovepress}

PubMed Central and CAS, and is the official journal of The Society of Clinical Ophthalmology (SCO). The manuscript management system is completely online and includes a very quick and fair peer-review system, which is all easy to use. Visit http://www.dovepress.com/ testimonials.php to read real quotes from published authors. 